\title{
LA SELVA INUNDABLE DE CANACOITE EN TABASCO, MÉXICO, UNA COMUNIDAD VEGETAL AMENAZADA
}

\author{
Emerson A. Maldonado-Sánchez ${ }^{1}$, Susana Ochoa-Gaona ${ }^{2,5}$, Rodimiro Ramos- \\ Reyes $^{3}$, María de los Ángeles Guadarrama-Olivera ${ }^{1}$, Noel González-Valdivia ${ }^{4}$ \\ Y BERNARdUS H. J. DE JONG ${ }^{2}$
}

${ }^{1}$ Universidad Juárez Autónoma de Tabasco, División Académica de Ciencias

Biológicas, Carretera Villahermosa-Cárdenas km. 0.5 s/n, 86150

Villahermosa, Tabasco, México.

${ }^{2}$ El Colegio de la Frontera Sur, Unidad Campeche, Departamento de Ciencias de la

Sustentabilidad, Av. Rancho Polígono 2-A, Ciudad Industrial,

Lerma, 24500 Campeche, Campeche, México.

${ }^{3}$ El Colegio de la Frontera Sur, Unidad Villahermosa, Laboratorio de Análisis

Geográfico, Carretera Villahermosa-Reforma km. 15.5, Ranchería

Guineo 2a sección, 86280 Villahermosa, Tabasco, México.

${ }^{4}$ Tecnológico Nacional de México, Departamento de Ingenierías, Instituto

Tecnológico de Chiná, Calle 11 s/n entre 22 y 28, 24520, Chiná,

Campeche, México.

${ }^{5}$ Autor para la correspondencia: sochoa@ecosur.mx

\section{RESUMEN}

Se analizó la estructura, composición y diversidad arbórea de los dos rodales más importantes de selva mediana perennifolia de Bravaisia integerrima (SMPC) de Tabasco, México: el de la Reserva Ecológica Yu-Balcah (YCAH) con una superficie de 271 ha y el del Parque Estatal La Chontalpa (PECH) con 191 ha. En cada uno se levantaron 10 parcelas de muestreo de $1000 \mathrm{~m}^{2}$, cubriendo un área total de 2 ha. Se identificó y registró a las plantas arbóreas, diferenciando brinzales, plantas juveniles y adultos. Se aplicaron diversos índices para analizar el esfuerzo de muestreo, la diversidad de especies, la abundancia proporcional, la dominancia y la equidad. El análisis estructural se basó en el valor de importancia. La riqueza total fue 76 especies, pertenecientes a 64 géneros y 31 familias botánicas, de las que la mejor representada fue Leguminosae con 15 especies. El área basal de YCAH y PECH fue de 37.2 y $29.5 \mathrm{~m}^{2} \mathrm{ha}^{-1}$, respectivamente. Bravaisia integerrima fue el árbol con mayor valor de importancia en los dos rodales con un Índice correspondiente de 277. Ambos fragmentos 
tienen un valor del índice de Shannon de 2.0. El sitio con mejor condición ecológica fue YCAH. Se identificaron seis especies catalogadas en la NOM-059-SEMARNAT-2010. La SMPC presenta una cobertura actual en Tabasco de 490 ha. Los resultados sugieren que su estructura y composición se han visto afectados por la actividad antrópica en la región. Para garantizar la conservación de los remanentes de SMPC en el estado, es importante revisar y actualizar su situación legal y sus planes de manejo, así como generar alternativas sociales y productivas en las poblaciones cercanas. Para lograrlo se requiere de la participación de actores gubernamentales, académicos, organizaciones no gubernamentales, particulares y sociedad en conjunto.

Palabras clave: bosques de Bravaisia, diversidad florística, reserva ecológica, riqueza arbórea.

\section{ABSTRACT}

The structure, composition and diversity of the floodplain forest (SMPC) of Bravaisia integerrima were studied in the two largest forest stands in Tabasco, Mexico: the Ecological Reserve Yu-Balcah (YCAH) with 271 ha, and State Park La Chontalpa (PECH) with 191 ha. In each forest stand 10 plots of $1000 \mathrm{~m}^{2}$ were sampled. Tree individuals sampled were identified to species, and evaluated in three age categories, seedlings, saplings, and adults. The relationship between species richness and sampling effort was analyzed using Chao 2, ICE, Jacknife 1, Jacknife 2 and Bootstrap. The structural analysis was based on the importance value index. The diversity of species was evaluated, using the Margalef index $\left(D_{M g}\right), \alpha$ de Fisher, Shannon-Wiener $\left(H^{\prime}\right)$ and equity of Pielou J. The dominance indices of Berger-Parker $(d)$ and Simpson $(D)$ were also used. The total number of species was 76 , belonging to 64 genera and 33 botanical families. The richest family was Leguminosae with 15 species. The basal area of the sites YCAH and PECH was 37.2 and $29.5 \mathrm{~m}^{2} \mathrm{ha}^{-1}$, respectively. Bravaisia integerrima was the most important tree species in all stands, with an Importance Index of 277. The value of the Shannon index was 2.0 in both sites. YCAH showed the best ecological condition. Six species present in the stands are considered as threatened in Mexico (NOM-059-SEMARNAT-2010). The current extent of the floodplain forest of Bravaisia in Tabasco State is 490 ha. The results suggest that the structure and composition of this forest has been affected by human activity. As a consequence, to guarantee the conservation of these remnants of SMPC in Tabasco, it is important to verify their legal situation and the application of management plans, designed to improve the productive activities of the rural communities that live around the stands. To succeed 
in this endeavor, it is necessary that governmental actors, academics, non-governmental organizations, and civil society work together.

Key words: arboreal richness, ecological reserve, tree diversity, tropical rain forest of Bravaisia.

\section{INTRODUCCIÓN}

En México, la selva tropical húmeda (selva alta y mediana perennifolia según Miranda y Hernández X., 1963; bosque tropical perennifolio según Rzedowski, 1978) llegó a ocupar la mayor parte del sur y sureste de México. Para 2007 se reporta que estas comunidades cubren $4.62 \%$ de la cobertura del territorio de la República (FRA, 2010), la cual se distribuye en numerosos fragmentos de lo que antes fuera una cobertura casi ininterrumpida (Challenger y Dirzo, 2009; Dirzo et al., 2009). Los trabajos sobre la estructura, diversidad, composición y el funcionamiento de la selva tropical húmeda han sido numerosos, entre ellos los de Bongers et al. (1988), Godínez-Ibarra y López-Mata (2002), De Walt y Chave (2004), Cortés-Castelán e Islebe (2005) y Martínez-Ramos y García-Orth (2007), por citar algunos. Sin embargo, estos estudios no diferencian las selvas que se presentan en condiciones de inundación en el sureste de México, como es el caso de la selva mediana perennifolia de canacoite (Bravaisia integerrima; SMPC; López-Mendoza, 1980). La SMPC se desarrolla sobre suelos arcillosos profundos con escaso drenaje, desde los 5 hasta los $140 \mathrm{~m}$ de altitud. Esta comunidad vegetal está constituida por diversas formas biológicas como hierbas, epifitas, enredaderas, arbustos y árboles. El dosel puede llegar hasta $25 \mathrm{~m}$ de altura con especies como zapote negro (Diospyros nigra), barí (Calophyllum brasiliense), chacahuanté (Simira salvadorensis) y tinco (Vatairea lundellii), con un sotobosque dominado por palmas espinosas como la palma chichón (Astrocaryum mexicanum), escoba (Cryosophila stauracantha) y el arbusto conocido como huesillo (Faramea occidentalis). Se distribuye sobre tierra firme inundable a lo largo de la vertiente del Pacífico desde Nayarit hasta Chiapas y en la del Atlántico como manchones puros o asociados a la selva alta perennifolia de pio (Licania platypus: Miranda y Hernández X., 1963; López-Mendoza, 1980; Pennington y Sarukhán, 2005). La pérdida de cobertura selvática se explica por los cambios de uso de suelo para la agricultura y ganadería, la extracción de madera, el crecimiento poblacional, el incremento de la infraestructura carretera y la actividad petrolera (Tudela, 1989; Salazar-Conde et al., 2004; Sánchez-Munguía, 2005; Gue- 
rra-Martínez y Ochoa-Gaona, 2008). En Tabasco la extensión original de SMPC se ha eliminado casi por completo y en la actualidad persisten solo escasos fragmentos de esta asociación vegetal. A pesar de conformar una comunidad ecológicamente relevante, en el estado ha sido poco estudiada teniendo como referencia los trabajos florísticos de Sol-Sánchez et al. (1999) y Maldonado-Sánchez y Maldonado-Mares (2010). El objetivo del presente trabajo fue generar información básica de la selva de canacoite para lo cual se describe su estructura, diversidad y composición arbórea, con el fin de resaltar su importancia en la cubierta vegetal del estado y sentar bases para su conservación y manejo a corto plazo.

\section{MATERIALES Y MÉTODOS}

Área de estudio.

Se estudiaron los dos fragmentos más grandes de selva mediana de canacoite en Tabasco. El primer sitio se ubica en el municipio de Cárdenas y pertenece al Parque Estatal La Chontalpa (PECH) con un rodal de selva de 191 ha, declarado Área Natural Protegida el 4 de junio de 1995 (Fig. 1A). Éste corresponde a la Subregión de La Chontalpa en la planicie fluvio-deltaica, que abarca la mayor parte del estado, la cual está formada por depósitos aluviales de carácter deltaico, con deficiente drenaje. Presenta suelos de texturas medias franco-arcillosas a arcillosas que corresponden al tipo Cambisol gleyico y Vertisol pélico (Palma y Cisneros, 1996; Inegi, 2000), mismos que se inundan durante la época de lluvias más intensas (septiembrenoviembre), por lo que están mal oxigenados. La vegetación predominante en la región fue de selva tropical húmeda inundable (López-Mendoza, 1980), asociada a amplias áreas cubiertas de vegetación de humedales emergentes de popal y tular principalmente. El clima en esta subregión es cálido húmedo (Am(f)w'(i')g), con temperatura media anual de $26{ }^{\circ} \mathrm{C}$, la media máxima mensual en mayo es de 30.3 ${ }^{\circ} \mathrm{C}$ y la media mínima en diciembre y enero de $20^{\circ} \mathrm{C}$. La precipitación en promedio anual es de $2463 \mathrm{~mm}$ (Cardoso, 1979).

El segundo sitio se ubica en la finca La Asunción en el ejido Ceibita del municipio de Tacotalpa. El lugar es un Área Natural Protegida de jurisdicción estatal denominada Reserva Ecológica Yu-Balcah (YCAH), declarada el 10 de junio del año 2000, con una superficie de 271 ha (Fig. 1B). Corresponde a la subregión de la Sierra que comprende parte de la unidad planicie fluvial interior del río La Sierra. Es de origen palustre con un nivel topográfico de alrededor de 50 m s.n.m., en una zona de transición regional sobre la cual se reconocen los escurrimientos que des- 

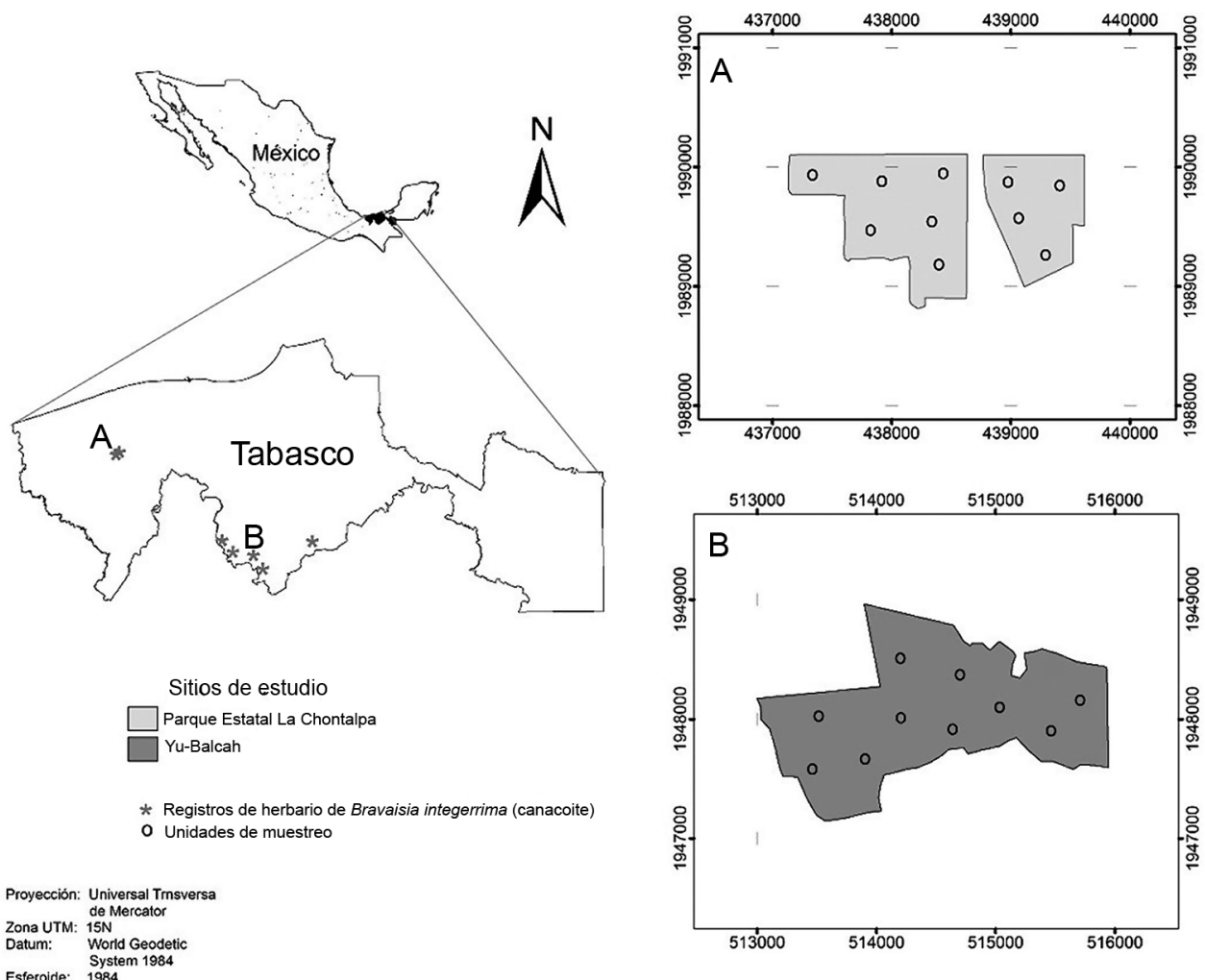

Sitios de estudio

$\square$ Parque Estatal La Chontalpa

Yu-Balcah

* Registros de herbario de Bravaisia integerrima (canacoite)

0 Unidades de muestreo

Esferoide: 1984

Fig. 1. Localización de los sitios de muestreo de selva mediana perennifolia de canacoite en Tabasco. Parque Estatal La Chontalpa $=$ PECH, Yu-Balcah $=$ YCAH.

cienden de las montañas del norte de Chiapas. Las unidades de suelo más comunes son Fluvisoles, Gleysoles y Vertisoles, dominados por arcilla y el manto freático se encuentra entre 1 y 2 m (Palma y Cisneros, 1996). En esta subregión el clima es cálido-húmedo con lluvias todo el año (Af(m)w"(i)g), con una temperatura media anual de $26^{\circ} \mathrm{C}$. La precipitación anual es de $4014 \mathrm{~mm}$ con un promedio máximo mensual de $588 \mathrm{~mm}$ en el mes de octubre y un mínimo mensual de $132 \mathrm{~mm}$ en el mes de abril (Inegi, 2000).

Selección de sitios de muestreo.

Para conocer los antecedentes de distribución de Bravaisia integerrima en Tabasco se revisaron las colectas de los herbarios CH (El Colegio de la Frontera 
Sur-San Cristóbal), UJAT (Universidad Juárez Autónoma de Tabasco), XAL (Instituto de Ecología, A.C.), CSAT (Colegio de Posgraduados-Tabasco Campus Tabasco), MEXU (Universidad Nacional Autónoma de México) y ENCB (Herbario de la Escuela Nacional de Ciencias Biológicas del Instituto Politécnico Nacional). Cada registro de herbario se georreferenció con base en la localidad anotada. Se realizaron recorridos para corroborar la presencia de la selva inundable de Bravaisia integerrima como elemento dominante, encontrando sólo tres fragmentos de la misma de canacoite en Tabasco los cuales no forman un continuo. Los dos mayores fueron los que se seleccionaron para el muestreo (YCAH y $\mathrm{PECH}$ ).

Caracterización de la vegetación.

En cada sitio se calculó la altura del dosel mediante una pértiga telescópica graduada de $18 \mathrm{~m}$ y un clinómetro Suunto. El diámetro de los árboles se midió a $1.30 \mathrm{~m}$ de altura (DAP), para canacoite $1.30 \mathrm{~m}$ por arriba de las raíces adventicias. El estado de conservación del sitio se determinó utilizando el índice-multicriterio para la evaluación de selvas tropicales (Ochoa-Gaona et al., 2010) que valora la condición ecológica del bosque. Este índice toma en cuenta la estructura vertical y horizontal, la regeneración de las especies arbóreas, la cubierta vegetal, la riqueza de especies, y factores de perturbación como árboles talados, muertos en pie o caídos, indicios de incendios, rastros de ganado, presencia de brechas y macheteo de vegetación.

Se establecieron de manera aleatoria 10 unidades de muestreo (UM) para YCAH y $10 \mathrm{UM}$ en PECH; las UM se separaron entre ellas al menos $50 \mathrm{~m}$. Las UM tuvieron forma de círculos concéntricos, adaptados de la metodología usada para inventarios forestales (SARH, 1994; Bechtold y Scott, 2005; PMC, 2011). Se identificaron y registraron todos los árboles y se midió el DAP (diámetro a la altura del pecho) de la siguiente manera: en el círculo de $1000 \mathrm{~m}^{2}$ (radio $\left.=17.84 \mathrm{~m}\right)$, de los de $\geq 20 \mathrm{~cm}$ de DAP; en el círculo intermedio de $500 \mathrm{~m}^{2}(\mathrm{r}=12.62 \mathrm{~m})$ de los individuos de $\geq 10 \mathrm{~cm}$ de DAP y en el círculo de $100 \mathrm{~m}^{2}(\mathrm{r}=5.64 \mathrm{~m})$ de $l o s$ de $\geq 5 \mathrm{~cm}$ DAP. Se consideraron adultos todos los árboles de $\geq 5 \mathrm{~cm}$ DAP. En el círculo de 100 $\mathrm{m}$ también se contabilizaron los individuos de $<5 \mathrm{~cm}$ DAP pero mayores de $1.30 \mathrm{~m}$ de altura (juveniles). Para el registro de brinzales y árboles juveniles tempranos de $<1.30 \mathrm{~m}$ de altura, se establecieron cuatro cuadros de $1 \mathrm{~m}^{2}$ por UM (Ochoa-Gaona et al., 2007). Se recolectaron muestras botánicas de cada especie arbórea registrada. La identificación se hizo mediante el corrimiento de claves y por comparación con ejemplares de herbario. Los especímenes herborizados fueron depositados en el herbario UJAT. 
Análisis de datos.

El incremento de la riqueza en función del esfuerzo de muestreo se analizó por medio de un método no paramétrico utilizando los estimadores Chao 2, ICE, Jacknife 1, Jacknife 2 y Bootstrap que usan datos de presencia-ausencia de especies (Leigh, 1999). El análisis estructural del bosque se basó en el valor de importancia (IVI) de cada especie por sitio, empleando los valores relativos de la densidad, frecuencia y el área basal $\left(\mathrm{IVI}=(\mathrm{DR}+\mathrm{FR}+\mathrm{ABR}) / 3\right.$. La densidad $\left(D\right.$; ind ha $\left.{ }^{-1}\right)$ es el número de individuos de la especie por unidad de superficie, para ello se tomó en cuenta el área estratificada en la que ésta fue muestreada la especie. La frecuencia (F) es el número de UM en las que se presenta una especie con relación a la cantidad total de UM. El área basal $\left(\mathrm{AB} ; \mathrm{m}^{2} \mathrm{ha}^{-1}\right)$ se obtuvo con la fórmula del área de un círculo con base en el DAP del tronco de cada individuo (Curtis y McIntosh 1951; Mueller-Dombois y Ellenberg 1974; Godínez-Ibarra y López-Mata 2002, GallardoCruz et al. 2005, Levy et al., 2006; Zamora-Crescencio et al., 2008).

Diversidad y similitud.

Para contar con elementos de comparación y análisis de la diversidad alfa en la SMPC, en cada UM (0.1 ha) y por sitio de estudio (1 ha) se calcularon los índices de riqueza específica como la riqueza de especies (S) y el índice de Margalef $\left(D_{M g}\right)$. Para lo estructural se usaron los índices de abundancia proporcional $\alpha$ de Fisher, Shannon-Wiener $\left(H^{\prime}\right)$ base logaritmo natural y equidad de Pielou J. Además se determinaron los índices de dominancia Berger-Parker $(d)$ y Simpson $(D$; Magurran, 1988). El cálculo de tales índices se realizó mediante el software libre Past 3.11 x Paleontological Statistics (Hammer, 1999-2015). Para efectos comparativos entre los dos sitios de estudio se utilizó el índice de diversidad Alfa de Fisher, obtenido mediante el software EstimateS (Colwell, 2013), que considera en su cálculo a la cantidad de especies (S) y al número total de individuos $(\mathrm{N})$ de las $\mathrm{UM}$, que al promediarse -siguiendo a López y Duque (2010)- conforman un estimador de la diversidad por hectárea, lo que hace posible comparar las comunidades entre los dos fragmentos. Este índice, además, es menos sensible al tamaño de muestreo que $H^{\prime}$ y no requiere unidades contiguas o de igual tamaño o forma, por lo que es más robusto y extrapolable (Leigh, 1999; Godínez-Ibarra y López-Mata, 2002; Ledo et al., 2012). Para analizar la variación en la estructura y diversidad taxonómica entre las parcelas, se realizó un análisis de componentes principales (PCA) que incluyó variables de estructura (número de individuos, área basal, promedio del diámetro a la altura del pecho), de diversidad (índices de Simpson, Shannon y de $\alpha$ de Fisher), así como taxonómicos (número de familias, géneros y especies). 


\section{RESULTADOS}

Riqueza y estructura. La curva de acumulación de especies en el sitio YCAH indica que es necesario un mayor esfuerzo de muestreo ya que se registró $61 \%$ (53 spp.) de la riqueza esperada ( 85 spp.) según el estimador de Chao 2 (Fig. 2A). Se contabilizaron allí 49 géneros y 24 familias. Las más representadas fueron Leguminosae con 10 especies, Moraceae y Rubiaceae con 5, y Malvaceae con 4. Se contaron 447 individuos, siendo más abundantes los de Astrocaryum mexicanum, Bravaisia integerrima, Cryosophila stauracantha y Guarea glabra. Los árboles con mayor frecuencia fueron Bravaisia integerrima, Guarea glabra, Cordia collococa, Spondias mombin y Licania platypus. El área basal promedio en YCAH fue de $37.2 \mathrm{~m}^{2} \mathrm{ha}^{-1}$. Con más altos valores de este parametro se registró a Bravaisia integerrima, Spondias mombin, Ceiba pentandra y Bursera simaruba. Las especies con mayor IVI en el sitio fueron Bravaisia integerrima, Astrocaryum mexicanum, Spondias mombin y Guarea glabra (Apéndice 1). YCAH presentó tres estratos arbóreos: el sotobosque con altura de hasta $10 \mathrm{~m}$, una capa media entre 10 y $18 \mathrm{~m}$ y uno superior con más de $18 \mathrm{~m}$. El sotobosque está dominado por palmas espinosas como Astrocaryum mexicanum, Cryosophila stauracantha y Bactris balanoidea. Otras especies acompañantes son Faramea occidentalis, Trichilia havanensis y Ampelocera hottlei. En el estrato medio Bravaisia integerrima es la dominante; con Cordia collococa, Andira inermis y Coccoloba barbadensis como acompañantes. El dosel alto presenta árboles de gran tamaño como Guarea glabra, Vatairea lundellii, Swietenia macrophylla, Ceiba pentandra, Calophyllum brasiliense, Dialium guianense y Diospyros nigra. Dentro de la selva se observaron claros de hasta $250 \mathrm{~m}^{2}$, principalmente en la zona de selva alta, producto de la caída natural de árboles. En ellos se registró reclutamiento (brinzales y plantas juveniles; Quinto-Mosquera et al., 2009) de especies de los tres estratos de la SMPC. En los claros se identificaron elementos de selva madura como Guatteria anomala, Guarea glabra, Vatairea lundellii y Diospyros nigra.

Para el sitio PECH en la curva de acumulación de especies se observó $79 \%$ (38 spp.) de la riqueza esperada (48 spp.) según el estimador de Chao 2 (Fig. 2B). Se inventariaron en el sitio 34 géneros y 24 familias. Las mejor representadas fueron Leguminosae con 10 especies, Moraceae con 5 y Rubiaceae con 3. En PECH se registraron 359 individuos; las especies más abundantes fueron Bravaisia integerrima, Faramea occidentalis, Tabernaemontana alba, Pleuranthodendron lindenii y Cordia collococa. Las más frecuentes eran Bravaisia integerrima, Cordia collococa, Pachira aquatica y Spondias mombin. El área basal promedio en PECH fue de $29.5 \mathrm{~m}^{2} \mathrm{ha}^{-1}$. Los árboles que aportaron mayor AB han sido Bravaisia integerrima, Ficus sp. 1, Spondias mombin y Guazuma ulmifolia. Las especies con mayor IVI en PECH fueron Bravaisia integerrima, Ficus 

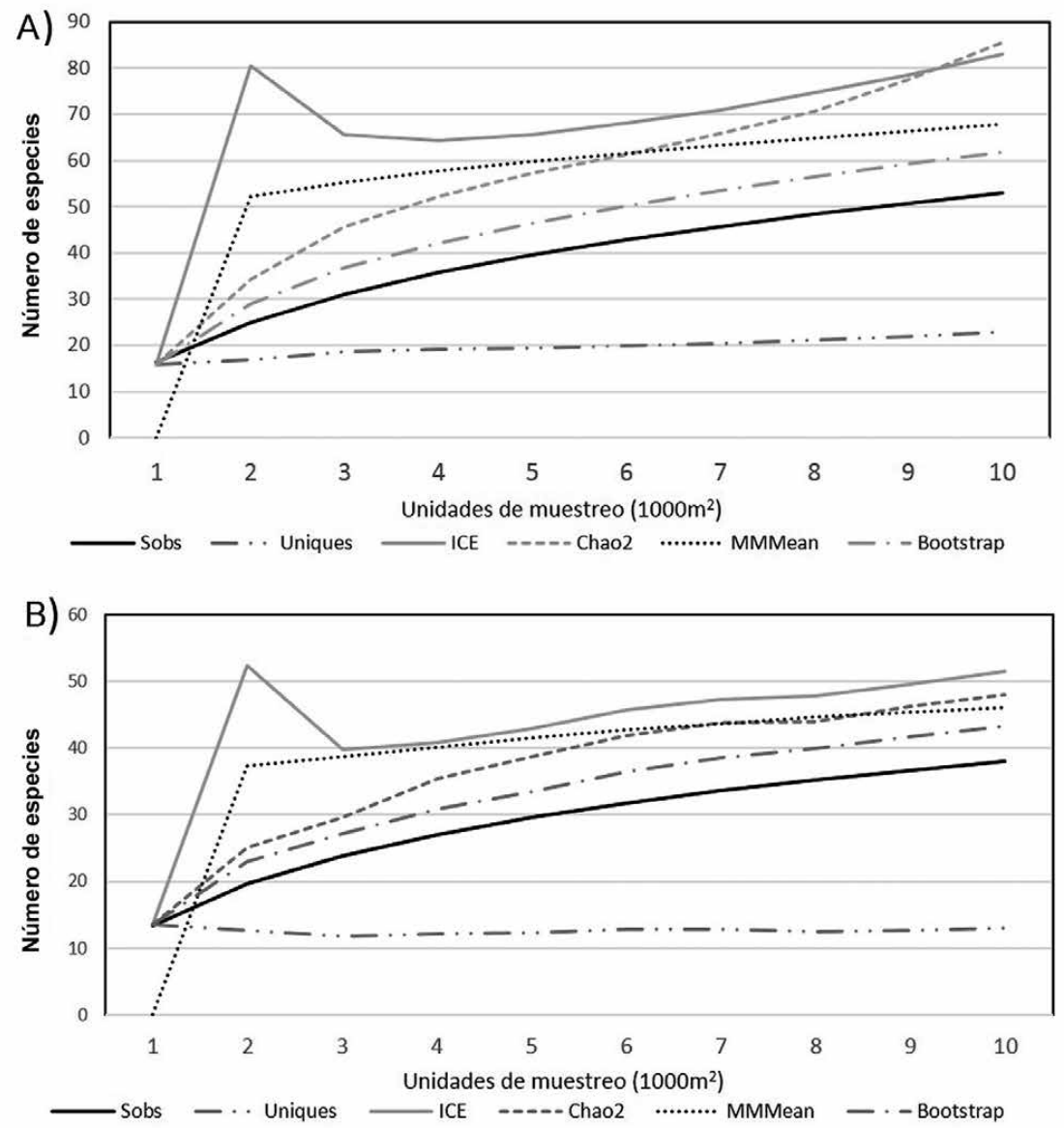

Fig. 2. Curvas de acumulación de especies en la SMPC de YCAH (A) y de PECH (B). En el eje $\mathrm{X}$ se muestra el esfuerzo de muestreo. El eje $\mathrm{Y}$ representa el número de especies observadas y estimadas para cada nivel de muestreo dado (Sn).

sp. 1, Spondias mombin y Faramea occidentalis. El bosque de PECH presentó tres estratos. En el bajo, entre los 3 y $8 \mathrm{~m}$ las de mayor densidad fueron Pleuranthodendron lindenii, Trichilia havanensis y Faramea occidentalis. El estrato medio alcanzó los $15 \mathrm{~m}$ y está dominado por árboles de Bravaisia integerrima, acompañados por Zygia cognata y Guazuma ulmifolia; el superior de 15 a $20 \mathrm{~m}$ forma el dosel y presenta a Spondias mombin, Ficus sp. 1, Bursera simaruba y Calophyllum brasiliense (Apéndice 2).

Las categorías diamétricas en los dos sitios obtuvieron una distribución de $\mathrm{J}$ invertida con numerosos individuos de clases pequeñas (Fig. 3). La mayoría de 


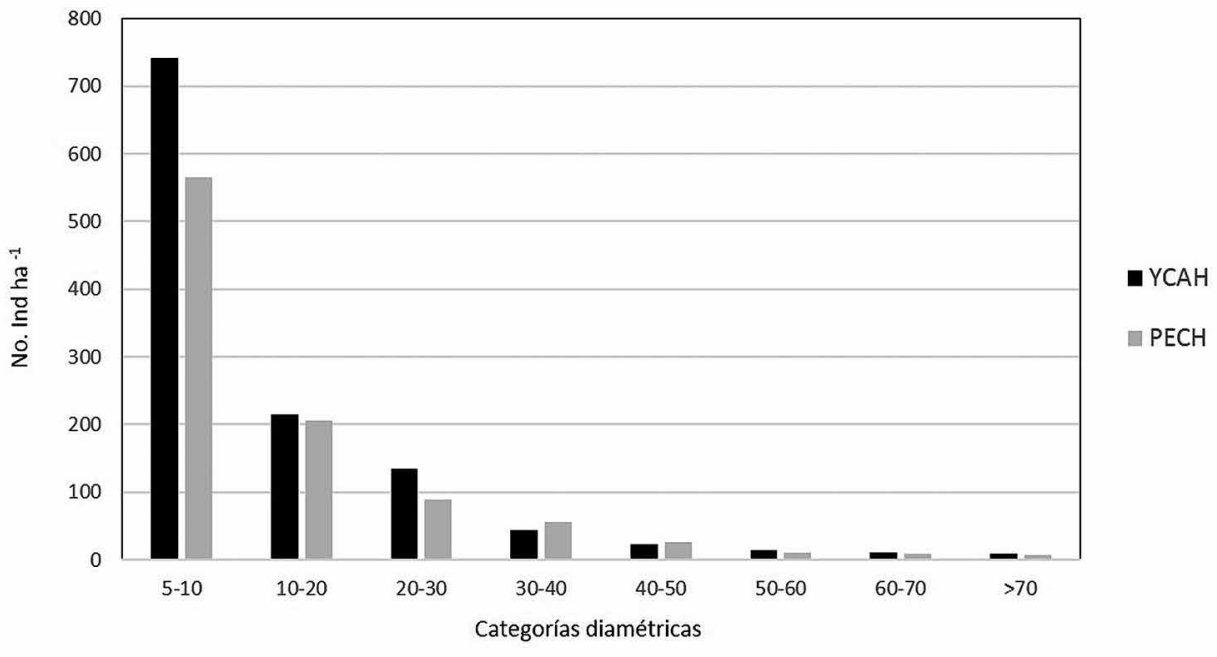

Fig. 3. Densidad de especies arbóreas de $\geq 5 \mathrm{~cm}$ DAP por categorías diamétricas de las selvas medianas perennifolias de canacoite en Tabasco.

las especies están representadas por pocos árboles, mientras que algunas registran abundancias altas. De las diez especies con mayor valor de importancia (IVI) en YCAH, Bravaisia integerrima, Astrocaryum mexicanum, Guarea glabra, Cryosophila stauracantha y Diospyros nigra contaron con individuos en los tres estadios de desarrollo (adultos, plantas juveniles, brinzales), mientras que Spondias mombin, Licania platypus, Vatairea lundellii y Guatteria anomala solo registraron árboles adultos (Fig. 4A). En el bosque de PECH las especies con mayor IVI que estuvieron presentes en los tres estadios de desarrollo fueron Bravaisia integerrima, Ficus sp. 2, Cordia collococca, Tabernaemontana alba, Pleuranthodendron lindenii y Zygia cognata mientras que solo se registraron adultos de Spondias mombin, Faramea occidentalis, Guazuma ulmifolia y Pachira aquatica (Fig. 4B).

Diversidad.

La riqueza a nivel de especie en YCAH fue de 53 especies y en PECH de 38, el promedio para los índices $\alpha$ de Fisher y de Margalef, así como el de Berger-Parker para evaluar dominancia resultaron ser mayores en YCAH, mientras que los índices de Shannon, de equidad de Pielou y Simpson fueron más altos en PECH (Cuadro 1).

Del total de especies registradas, $26 \%$ se comparten entre los dos sitios, las cuales en orden decreciente de IVI son: Bravaisia integerrima, Spondias mombin, Ficus 

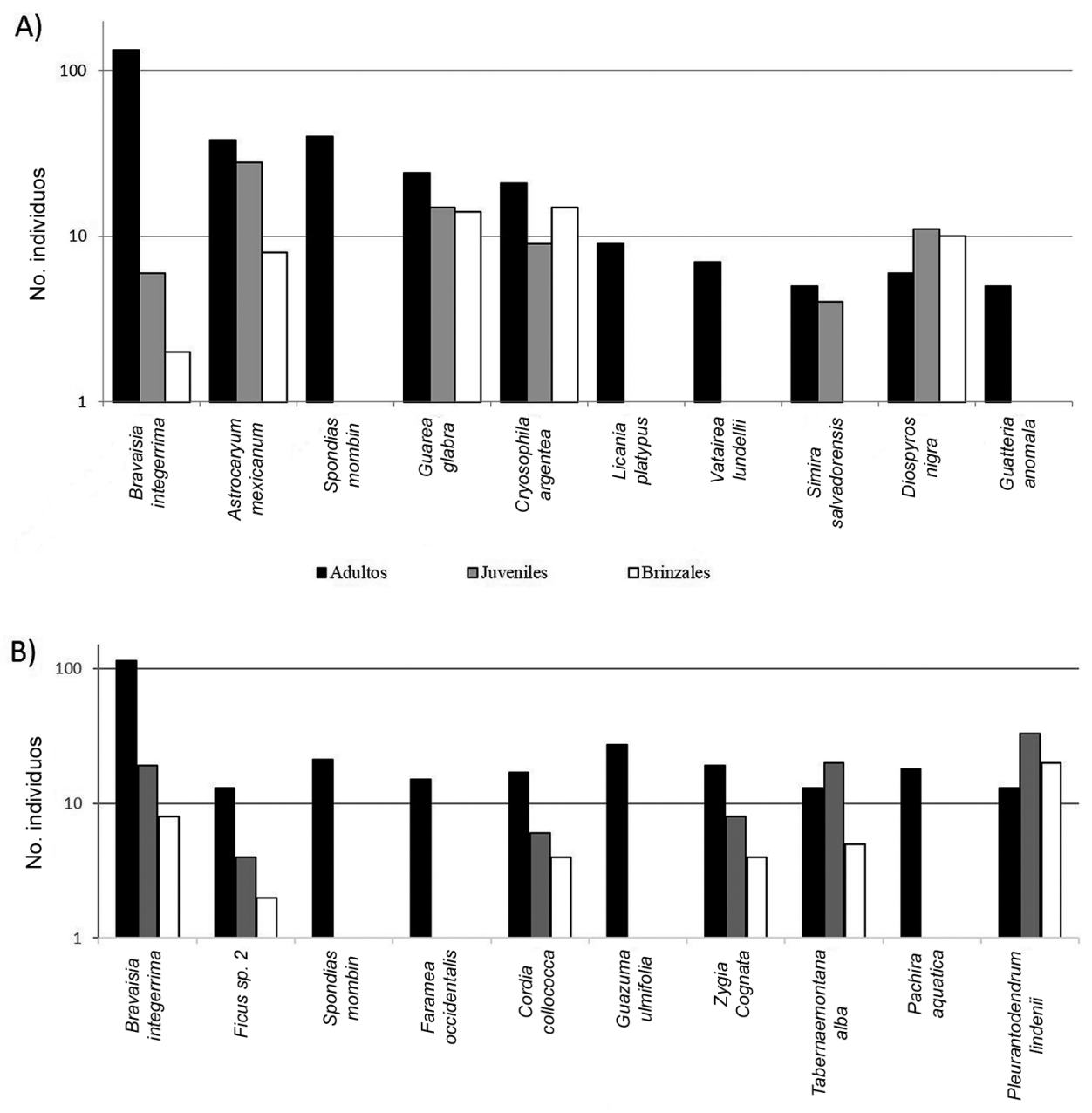

- Adultos a Juveniles aBrinzales

Fig. 4. Repoblación para las diez especies con mayor valor de importancia (IVI) en YCAH (A) y Pech (B).

sp. 1, Zygia cognata, Pachira aquatica, Tabernaemontana alba, Calophyllum brasiliense, Coccoloba barbadensis, Faramea occidentalis, Trophis racemosa, Licania platypus, Andira inermis, Casearia corymbosa, Cecropia obtusifolia y Tabebuia rosea.

Se encontró que el YCAH presenta mejor condición ecológica (valores más cercanos a 1) que PECH (Cuadro 2). 
Cuadro 1. Valores de riqueza, índices de diversidad y dominancia calculados para los dos sitios de selva inundable de canacoite. $\mathrm{UM}=$ Unidades de muestreo, $\mathrm{S}=$ Riqueza específica, $\alpha=$ Alfa de Fisher, $D_{M g}=$ Margalef, $H^{\prime}=$ Shannon-Wiener, $J=$ Pielou, $d=$ Berger-Parker, $D$ $=$ Simpson. d.e. $=$ desviación estándar.

\begin{tabular}{|c|c|c|c|c|c|c|c|}
\hline UM & $\mathrm{S}$ & $\alpha$ & $D_{M g}$ & $H^{\prime}$ & $J$ & $d$ & $D$ \\
\hline YCAH-1 & 15 & 6.49 & 3.43 & 2.22 & 0.82 & 0.36 & 0.83 \\
\hline YCAH-2 & 14 & 3.56 & 2.51 & 1.16 & 0.44 & 0.73 & 0.46 \\
\hline YCAH-3 & 17 & 3.95 & 2.83 & 2.05 & 0.73 & 0.42 & 0.79 \\
\hline YCAH-4 & 12 & 2.96 & 2.15 & 1.74 & 0.70 & 0.41 & 0.75 \\
\hline YCAH-5 & 17 & 6.39 & 3.60 & 2.23 & 0.79 & 0.34 & 0.83 \\
\hline YCAH-6 & 15 & 5.35 & 3.17 & 2.03 & 0.75 & 0.36 & 0.81 \\
\hline YCAH-7 & 16 & 5.43 & 3.27 & 1.81 & 0.65 & 0.41 & 0.74 \\
\hline YCAH-8 & 17 & 6.86 & 3.71 & 2.08 & 0.73 & 0.32 & 0.81 \\
\hline YCAH-9 & 17 & 6.75 & 3.68 & 2.32 & 0.82 & 0.26 & 0.86 \\
\hline YCAH-10 & 24 & 10.27 & 5.04 & 2.25 & 0.71 & 0.42 & 0.79 \\
\hline Promedio \pm d.e. & & $5.8 \pm 2.09$ & $3.3 \pm 0.79$ & $1.98 \pm 0.34$ & $0.71 \pm 0.11$ & $0.40 \pm 0.12$ & $0.76 \pm 0.11$ \\
\hline PECH-1 & 10 & 3.21 & 2.13 & 1.45 & 0.63 & 0.62 & 0.59 \\
\hline PECH-2 & 11 & 2.65 & 1.96 & 1.95 & 0.81 & 0.36 & 0.80 \\
\hline PECH-3 & 17 & 5.39 & 3.34 & 2.18 & 0.77 & 0.22 & 0.85 \\
\hline PECH-4 & 15 & 4.44 & 2.90 & 2.17 & 0.80 & 0.23 & 0.86 \\
\hline PECH-5 & 12 & 4.69 & 2.73 & 1.86 & 0.75 & 0.43 & 0.76 \\
\hline PECH-6 & 7 & 2.72 & 1.72 & 1.72 & 0.88 & 0.30 & 0.79 \\
\hline PECH-7 & 17 & 5.51 & 3.37 & 2.57 & 0.91 & 0.17 & 0.91 \\
\hline PECH-8 & 17 & 5.07 & 3.24 & 2.31 & 0.81 & 0.23 & 0.87 \\
\hline PECH-9 & 12 & 3.92 & 2.51 & 2.10 & 0.84 & 0.26 & 0.85 \\
\hline PECH-10 & 16 & 6.48 & 3.53 & 2.17 & 0.78 & 0.29 & 0.84 \\
\hline Promedio \pm d.e. & & $4.40 \pm 1.27$ & $2.7 \pm 0.64$ & $2.04 \pm 0.31$ & $0.79 \pm 0.07$ & $0.31 \pm 0.13$ & $0.81 \pm 0.08$ \\
\hline
\end{tabular}

En total se registraron seis especies catalogadas en alguna categoría de riesgo de la NOM-059-SEMARNAT-2010: Bravaisia integerrima (Spreng.) Standl, Calophyllum brasiliense Cambess., Cryosophila stauracantha (Heynh.) R.J. Evans, Guatteria anomala R.E. Fr., Ormosia macrocalyx Ducke, Vatairea lundellii (Standl.) Record, de las cuales cuatro se encuentran Amenazadas y dos en Peligro de Extinción (Semarnat, 2010). En YCAH se encontraron las seis especies y en PECH sólo tres de ellas (Apéndices 1 y 2).

El análisis multivariado indica que el eje 1 explica 55\% de la variación de los datos, y el eje $221 \%$. Las variables de diversidad y taxonómicas, así como área basal se 
Cuadro 2. Valor de condición ecológica de los sitios remanentes de SMPC en Tabasco. Se mide de 0 a 1 , siendo 1 el de mejor estado de conservación

\begin{tabular}{ccc}
\hline Unidad de & \multicolumn{2}{c}{ Sitio } \\
\cline { 2 - 3 } muestreo & YCAH & PECH \\
\hline 1 & 0.891 & 0.869 \\
2 & 0.889 & 0.831 \\
3 & 0.761 & 0.753 \\
4 & 0.931 & 0.841 \\
5 & 0.911 & 0.878 \\
6 & 0.932 & 0.741 \\
7 & 0.937 & 0.859 \\
8 & 0.959 & 0.669 \\
9 & 0.784 & 0.713 \\
10 & 0.927 & 0.553 \\
\hline Promedio & 0.8922 & 0.7707 \\
\hline
\end{tabular}

relacionan significativamente con el componente 1 del PCA, mientras que el número de individuos, y el índice de dominancia de Simpson están asociados con el componente 2 (Cuadro 3). Las UM que se ubican hacia el lado inferior de la ordenación están principalmente relacionadas con el eje 1, las UM localizadas en la parte superior del cuadrante están relacionadas positivamente con el eje 2 (Fig. 5). Se observa que varias UM están compartidas hacia la zona media, y algunas UM de ambos sitios se ubican como extremas. Esas son las que causan la variación y reflejan el posible efecto de la perturbación que tiene cada fragmento.

Cuadro 3. Coeficientes de correlación de Spearman obtenidos mediante PCA tras relacionar los valores estructurales y de diversidad de la selva inundable de canacoite con los puntajes de cada uno de los ejes del análisis de componentes principales. $\mathrm{n}=20 ; *=\mathrm{p} \leq 0.05$. Porcentaje de varianza: por Eje $1=55 \%$ y Eje $2=21 \%$.

\begin{tabular}{lccc}
\hline Variable & Acrónimo & Eje 1 & Eje 2 \\
\hline Número de individuos & NI & -0.0195 & $0.8181^{*}$ \\
Diámetro a la altura del pecho & DAP & 0.4513 & $-0.6490^{*}$ \\
Área basal & AB & $0.8161^{*}$ & 0.1104 \\
Índice de Simpson & D & 0.5462 & -0.5313
\end{tabular}


Cuadro 3. Continuación

\begin{tabular}{lccc}
\hline Variable & Acrónimo & Eje 1 & Eje 2 \\
\hline Índice de Shannon & $\mathrm{H}$ & $0.7655^{*}$ & -0.3465 \\
Índice $\alpha$ de Fisher & $\mathrm{F}$ & $0.8866^{*}$ & -0.1183 \\
Número de familias & $\mathrm{NF}$ & $0.8396^{*}$ & 0.3688 \\
Número de géneros & $\mathrm{NG}$ & $0.9195^{*}$ & 0.3278 \\
Número de especies & $\mathrm{NE}$ & $0.9263^{*}$ & 0.2894 \\
\hline
\end{tabular}

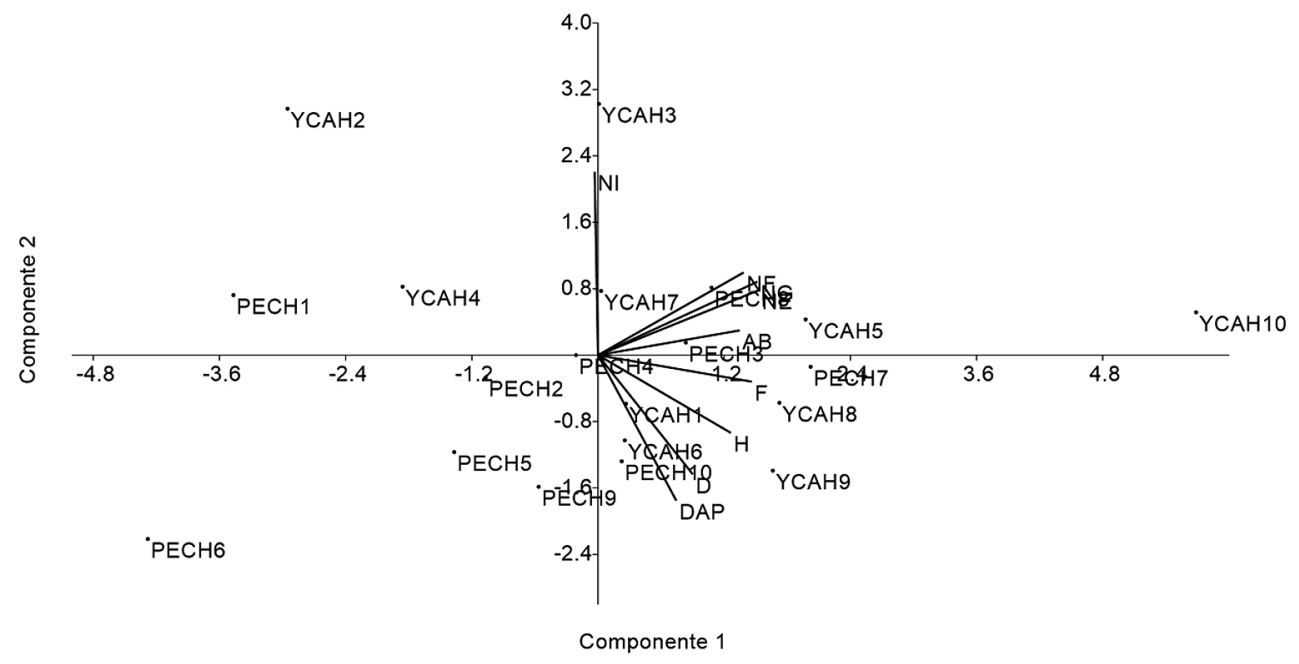

Fig. 5. Análisis de componentes principales (PCA) de los atributos estructurales y de diversidad de la comunidad arbórea de la selva mediana perennifolia de canacoite. Número de individuos (NI), diámetro a la altura del pecho (DAP), área basal (AB), Simpson $(D)$, Shannon $\left(H^{\prime}\right), \alpha$ de Fisher (F), número de familias (NF), número de géneros $(\mathrm{NG})$, número de especies (NE).

\section{DISCUSIÓN}

La selva mediana perennifolia de canacoite (SMPC) representa una de las comunidades vegetales más reducidas en extensión a nivel nacional con una distribución restringida a algunas zonas próximas a las costas del Pacífico y Golfo de México (Palacios, 2006), lo cual se corroboró con la revisión de herbario realizada en el presente estudio. Esta comunidad vegetal está limitada a áreas con suelos que se saturan de agua, lo cual es un requerimiento de Bravaisia integerrima que es 
su especie característica (Pennington y Sarukhán, 2005). La comunidad tiene gran importancia ecológica, ya que además de influir en el ciclo del agua, funciona como hábitat de fauna y contiene alta riqueza florística (De Grandi et al., 2000, Lou y Yurrita, 2005). Sin embargo, debido al acelerado proceso de deforestación, en el estado de Tabasco solo persisten tres remanentes con cierta condición original, los cuales en conjunto no alcanzan las 500 ha de superficie. La SMPC estudiada está dominada por Bravaisia integerrima (Amenazada, NOM-059-SEMARNAT-2010), árbol con grandes raíces adventicias y gran abundancia de palmas espinosas, lo que la define como una comunidad con condiciones biológico-ambientales particulares. A pesar de ello, ha recibido poca atención en su conocimiento, manejo y conservación, y actualmente estos remanentes están por desaparecer ya que se encuentran aislados y sometidos a gran presión antrópica (Ramos-Reyes et al., 2011).

\section{Riqueza y estructura.}

La cantidad de especies, al igual que de familias y géneros reportados en este trabajo, concuerdan con la encontrada en algunos bosques inundables de México (Díaz-Gallegos et al., 2002; Cortés-Castelan e Islebe, 2005; Zamora-Crescencio et al., 2008) y América tropical (Díaz y Rosales, 2006; Guerra y Pietrangeli, 2007; Díaz-Pérez et al., 2012). En lo local, Sol-Sánchez et al. (1999) y Maldonado-Sánchez y Maldonado-Mares (2010) registraron 83 y 51 especies de árboles respectivamente en selvas de las mismas características que la de este estudio, pero con diferencias metodológicas, como la inlcusión de individuos de DAP de $>1 \mathrm{~cm}$ de los primeros autores y DAP de $>5 \mathrm{~cm}$ usada por los segundos, ambos con superficie menor de muestreo que las del presente trabajo. Sin embargo, el medir individuos de $\geq 5 \mathrm{~cm}$ de DAP y muestrear 10 unidades de 0.1 ha en cada sitio, aún resultaron insuficientes por las características de heterogeneidad en el sistema.

El remanente de $\mathrm{PECH}$ presentó menor riqueza que $\mathrm{YCAH}$, lo cual puede deberse al mayor disturbio humano tal como lo demuestra la evaluación mediante el índice de condición ecológica aplicado (Ochoa-Gaona et al. 2010). Esto mismo se corrobora con los bajos valores para el índice alfa de Fisher $(<7)$ de nuestras parcelas, especialmente si se compara con la amplitud (11 a 211) que Godínez-Ibarra y López-Mata (2002) reportan para estudios realizados en diez selvas neotropicales de América.

La selva mediana perennifolia de canacoite estudiada contiene especies como Astrocaryum mexicanum, Bravaisia integerrima, Diospyros nigra, Calophyllum brasiliense, Faramea occidentalis, Simira salvadorensis, Vatairea lundellii y Licania platypus, las cuales han sido reportadas como características de selvas maduras 
perennifolias (Miranda y Hernández X., 1963; Bongers et al., 1987; López-Mendoza, 1980; Pennington y Sarukhán, 2005). Éstas, junto con Guarea glabra, han sido citadas en otros trabajos en selvas inundables (Pérez y Sarukhán, 1970; LópezMendoza, 1980; Ochoa-Gaona y Domínguez-Vázquez, 2000; Levy et al., 2006), lo cual nos indica que los sitios estudiados aún conservan elementos característicos y propios de bosques poco alterados, hecho que resalta la importancia de su conservación, en especial por el gran avance de deforestación en el estado.

Las especies con mayor valor de importancia en la SMPC fueron Bravaisia integerrima, Spondias mombin, Cordia collococa y Astrocaryum mexicanum; mismas que también fueron reportadas con alto IVI por López-Mendoza (1980); Bongers et al. (1988) y Sol-Sánchez et al. (1999).

En YCAH se observa una convivencia única en Tabasco, entre selva mediana perennifolia de Bravaisia integerrima con selva alta perennifolia de Licania platypus (López-Mendoza, 1980); aunque actualmente la dominancia de L. platypus ha sido sustituida por otros árboles como Spondias mombin y Simira salvadorensis. Este tipo de asociación, así como la distribución de sus especies, está determinada posiblemente por la existencia de áreas con suelos inundables y otras no inundables, así como por diferencias en la profundidad y el tipo de suelo (Sollins, 1998; CortésCastelán y Islebe, 2005). El fragmento de SMPC de YCAH presentó las mejores características de conservación según el índice de condición ecológica aplicado, lo cual se corrobora por la mayor diversidad de especies, por la presencia de adultos y brinzales de elementos de selva madura, y por sus características fisonómicas y estructurales bajo condición de inundación que caracteriza a esta comunidad. El sitio YCAH tiene mayor dificultad de acceso, menor cercanía a núcleos poblacionales y carreteras, bajo desarrollo de la actividad agrícola y ganadera circundante y no tanta actividad petrolera en la región; todo lo anterior se traduce en una menor intensidad de disturbio humano al interior de este fragmento, conservando una mejor condición ecológica. Por el contrario, los factores antes mencionados afectan al sitio $\mathrm{PECH}$, lo que pone en riesgo la permanencia de este remanente de selva de canacoite en la Chontalpa. Por su parte, PECH presenta menor diversidad y condición ecológica más baja; esto puede deberse a la tala selectiva de maderas preciosas y útiles para la construcción por los pobladores de las zonas adyacentes a esta selva.

El promedio de individuos con DAP de $\geq 5 \mathrm{~cm}$ encontrados en la selva inundable de canacoite fue de 356 ind ha ${ }^{-1}$, con numerosos árboles de troncos de diámetros pequeños y pocos de tallos mayores de $50 \mathrm{~cm}$ o más de DAP, característicos de selvas tropicales del sureste de México (Tun-Dzul et al., 2008; Zarco-Espinoza et al., 2010; Vázquez-Negrín et al., 2011). La densidad de individuos arbóreos fue 
inferior a los 420 ind ha-1 reportados por Balslev et al. (1987) en Añangu, Ecuador y similar a lo registrado por Lieberman et al. (1985) y Johnston y Gillman (1995) en bosques inundables de tierras bajas en Costa Rica y Guyana (353 y 357 ind ha ${ }^{1}$ respectivamente). En los sitios estudiados se observó baja cantidad de árboles adultos de especies típicas de selva madura, lo que acrecienta el riesgo de que junto con la tala inmoderada y la pequeña área que ocupan los fragmentos de la selva inundable en Tabasco, se incrementen las posibilidades de extinción local de las especies (Finegan y Bouroncle, 2008). El área basal promedio encontrada en la selva de canacoite fue de $33.34 \mathrm{~m}^{2}$ ha-1, y es similar a lo encontrado en otros estudios realizados en selvas conservadas como las medianas de Tabasco, Veracruz y Chiapas (Pérez y Sarukhán, 1970; Sol-Sánchez et al., 1999; Godínez-Ibarra y López-Mata, 2002; Basáñez et al., 2008; Zarco-Espinoza et al. 2010), los bosques tropicales de Centroamérica (Cascante y Estrada, 2001; Chave et al., 2003; De Walt y Chave, 2004), en Malasia (Manokaran y LaFrankie, 1990), y de Camerún (van Gemerden et al., 2004), cuyos valores de área basal oscilan entre 21.2 y $45.3 \mathrm{~m}^{2} \mathrm{ha}^{-1}$. En conclusión, los remanentes estudiados aún conservan las características estructurales propias de selvas maduras a pesar de la intensa fragmentación y de estar inmersos en medio de extensas áreas agropecuarias, así como por lo reducido del área que ocupan.

Diversidad de especies.

El valor de diversidad de Shannon-Wiener $\left(H^{\prime}\right)$ obtenido para la SMPC y en particular en YCAH fue $\geq 2.0$, el cual se considera alto con base en las categorías propuestas por Ramírez-González (2006), así como por los números reportados para selvas tropicales del sureste de México (Durán, 1995; Sol-Sánchez et al., 1999; DíazGallegos et al., 2002; Godínez-Ibarra y López-Mata, 2002; Pérez et al., 2005). En este mismo sentido, el índice de diversidad de Margalef de las parcelas muestreadas y de los remanentes en su conjunto fue mayor a 2.0, que es considerado como de mediana a alta diversidad, e incluso la parcela YCAH-10 mostró un valor superior a 5.0, que es valorado como un indicador de muy elevada biodiversidad (OrellanaLara, 2009). El área basal en los dos sitios estuvo dominada por Bravaisia integerrima; esto se puede explicar por la alta biomasa evidenciada por sus troncos más gruesos, así también por su alta abundancia en los sitios. En la SMPC se observó la presencia de diversas formas biológicas tales como hierbas y bejucos, las cuales no fueron muestreadas y que incrementarían los valores de riqueza y diversidad de las comunidades estudiadas.

El registro de seis especies vegetales catalogadas en la NOM-059SEMARNAT-2010, Bravaisia integerrima, Calophyllum brasiliense, Cryosophila 
stauracantha, Guatteria anomala, Ormosia macrocalyx y Vatairea lundellii, demuestra la importancia ecológica de los fragmentos aislados de SMPC remanentes. Las acciones de conservación legal de estos remanentes de SMPC han resultado en su mantenimiento; sin embargo, está lejos de ser la solución a la pérdida de diversidad genética de este tipo de comunidades. Para garantizar la conservación de los remanentes de SMPC en Tabasco, es importante revisar y actualizar su situación legal, sus planes de manejo y su aplicación, llevar a cabo alternativas de conservación que incluyan aspectos como investigación básica y aplicada referente a su biodiversidad y manejo, así como que se faciliten alternativas sociales y productivas a las poblaciones cercanas. Para lograrlo se requiere de la participación de actores como gobierno, sector académico, organizaciones no gubernamentales, particulares y sociedad en conjunto.

\section{AGRADECIMIENTOS}

Agradecemos a Nelly del Carmen Jiménez Pérez por su valiosa ayuda en la identificación de las especies. A los guías de campo Don Juan y Antonio Alvarado. A los herbarios CH, UJAT, XAL, CSAT, MEXU y ENCB que permitieron el acceso a sus colecciones. A Fondos Mixtos del Consejo Nacional de Ciencia y Tecnología-Tabasco que financiaron el trabajo bajo el proyecto "Composición, estructura y distribución de selva mediana inundable de Bravaisia integerrima (Spreng.) Standl. en el estado de Tabasco, México" con número de registro TAB-2009-C16-121006. Al Consejo Nacional de Ciencia y Tecnología por la beca otorgada No. 32578 al primer autor para sus estudios de posgrado. A El Colegio de la Frontera Sur por el apoyo en infraestructura y asistencia académica. Finalmente a los árbitros revisores por su tiempo y dedicación.

\section{LITERATURA CITADA}

Balslev, H., J. L. Luteyn, B. Øllgaard y L. B. Holm-Nielsen. 1987. Composition and structure of adjacent unflooded and floodplain forest in Amazonian Ecuador. Opera Bot. 92: $37-57$.

Basáñez, A. J., J. L. Alanis y E. Badillo. 2008. Composición florística y estructura arbórea de la selva mediana subperennifolia del ejido "El Remolino", Papantla, Veracruz. Avances en Investigación Agropecuaria 12: 3-21.

Bechtold, W. A. y C. T. Scott. 2005. The forest inventory and analysis plot design. In: Bechtold W. A. y P. L. Patterson (eds.). The enhanced forest inventory and analysis 
program-national sampling design and estimation procedures. General Technical Report SRS-80 Forest Service, United States Department of Agriculture. Asheville, USA. pp. 27-42.

Bongers, F., J. Popma, J. Meave del Castillo y J. Carabias. 1988. Structure and floristic composition of the lowland rain forest of Los Tuxtlas, México. Vegetatio 74: $55-80$.

Cardoso, D. M. 1979. El clima de Chiapas y Tabasco. Instituto de Geografía. Universidad Nacional Autónoma de México. México D.F., México. 99 pp.

Cascante, A. y A. Estrada. 2001. Composición florística y estructura de un bosque húmedo premontano en el Valle Central de Costa Rica. Rev. Biol. Trop. 49: 213-225.

Challenger, A. y R. Dirzo. 2009. Tendencias de cambio y estado de la biodiversidad, los ecosistemas y sus servicios. In: Sarukhán J., R. Dirzo, R. González y I. J. March (eds.). Estado de conservación y tendencias de cambio. Capital Natural de México. Comisión Nacional para el Conocimiento y Uso de la Biodiversidad. México, D.F., México. pp. 35-72.

Chave, J., R. Condit, S. Lao, J. P. Caspersen, R. B. Foster y S. P. Hubbell. 2003. Spatial and temporal variation of biomass in a tropical forest: results from a large census plot in Panama. J. Ecol. 91: 240-252.

Colwell, R. K. 2013. EstimateS 9.1.0 User's Guide. http://viceroy.eeb.uconn.edu/estimates/ EstimateSPages/EstSUsersGuide/EstimateSUsersGuide.htm (consultado el 22 de febrero de 2016).

Cortés-Castelán, J. y G. A. Islebe. 2005. Influencia de los factores ambientales en la distribución de especies arbóreas en las selvas del sureste de México. Rev. Biol. Trop. 53: $1-24$.

Curtis, J. T. y R. P. McIntosh. 1951. An upland forest continuum in the prairie-forest border region of Wisconsin. Ecology 32: 63-81.

De Grandi, G. F., P. Mayaux, J. P. Malingreau, A. Rosenqvist, S. Saatchi y M. Simard. 2000. New perspectives on global ecosystems from wide-area radar mosaics: flooded forest mapping in the tropics. Int. J. Remote Sens. 21: 1235-1249.

De Walt, S. J. y J. Chave. 2004. Structure and biomass of four lowland Neotropical forests. Biotropica 36: 7-19.

Díaz, W. y J. Rosales. 2006. Análisis florístico y descripción de la vegetación inundable de várzeas Orinoquenses en el Bajo Río Orinoco, Venezuela. Acta Bot. Venez. 29(1): $39-68$.

Díaz-Gallegos, J. R., O. Castillo y G. García. 2002. Distribución espacial y estructura de una selva baja en un ejido de la Reserva de la Biosfera Calakmul, Campeche. Universidad y Ciencia 18: 9-26.

Díaz-Pérez, W. A., F. Daza y W. Sarmiento. 2012. Composición florística, estructura y diversidad del bosque ribereño del Río Kakada, Cuenca del Río Caura, estado Bolívar, Venezuela. Rev. Cient. UDO Agrícola 12(2): 275-289.

Dirzo, R., A. Aguirre y J. C. López. 2009. Diversidad florística de las selvas húmedas en paisajes antropizados. Investigación Ambiental 1: 17-22.

Durán, G. R. 1995. Diversidad florística de los Petenes de Campeche. Acta Bot. Mex. 31: 73-84. 
Finegan, B. y C. Bouroncle. 2008. Patrones de fragmentación de los bosques de tierras bajas, su impacto en las comunidades y especies vegetales y propuestas para su mitigación. In: Harvey, C. y J. Sáenz (eds.). Evaluación y conservación de biodiversidad en paisajes fragmentados en Mesoamérica. Instituto Nacional de Biodiversidad. San José, Costa Rica. pp. 139-178.

FRA. 2010. Evaluación de los recursos forestales mundiales 2010. Informe Nacional México. Evaluación de los Recursos Forestales Mundiales (FRA). Departamento Forestal, Organización de las Naciones Unidas para la Agricultura y la Alimentación. Roma, Italia. 98 pp.

Gallardo-Cruz, J. A., J. A. Meave y E. A. Pérez-García. 2005. Estructura, composición y diversidad de la selva baja caducifolia del Cerro Verde, Nizanda (Oaxaca), México. Bol. Soc. Bot. Méx. 76: 19-35.

Godínez-Ibarra, O. y L. López-Mata. 2002. Estructura, composición, riqueza y diversidad de árboles en tres muestras de selva mediana subperennifolia. Anales Inst. Biol. Serie Bot. 73: 283-314.

Guerra, M. y M. Pietrangeli. 2007. Caracterización florística de las comunidades forestales ribereñas presentes en un sector de la cuenca media del Río Socuy, estado Zulia, Venezuela. Rev. Fac. Agron. 24: 427-434.

Guerra-Martínez, V. y S. Ochoa-Gaona. 2008. Evaluación del programa de manejo de la Reserva de la Biosfera Pantanos de Centla, Tabasco, México. Universidad y Ciencia 24(2): 135-146.

Hammer, Ø. 1999-2015. Past: PAleontolgical STatistics V 3.11. Reference Manual. Natural History Museum. University of Oslo. http://folk.uio.no/ohammer/past/past3manual. pdf (consultado el 22 de febrero de 2015).

Inegi. 2000. Síntesis geográfica, nomenclator y anexo cartográfico del estado de Tabasco. Instituto Nacional de Estadística y Geografía. Aguascalientes, México. 116 pp.

Johnston, M. y M. Gillman.1995. Tree population studies in low-diversity forest, Guyana. Floristic composition and stand structure. Biodivers. Conserv. 4: 339-362.

Ledo, A., S. Condés y F. Montes. 2012. Revisión de índices de distribución espacial usados en inventarios forestales y su aplicación en bosques tropicales. Rev. Peru Biol. 19: 113-124.

Leigh, E. G. Jr. 1999. Tropical diversity. In: Leigh Jr. E. G. (ed.). Tropical forest ecology: A view from Barro Colorado Island. Oxford University Press. New York, USA. pp. 179-210.

Levy, T. S., R. R. Aguirre, P. J. García y R. M. Martínez. 2006. Aspectos florísticos de Lacanhá Chansayab, Selva Lacandona, Chiapas. Acta Bot. Mex. 77: 69-98.

Lieberman, M., D. Lieberman, G. S. Hartshorn y R. Peralta. 1985. Small-scale altitudinal variation in lowland wet tropical forest vegetation. J. Ecol. 73: 505-516.

López, W. y A. Duque. 2010. Patrones de diversidad alfa en tres fragmentos de bosques montanos en la región norte de los Andes, Colombia. Rev. Biol. Trop. 58: 483-498.

López-Mendoza, R. 1980. Tipos de vegetación y su distribución en el estado de Tabasco y norte de Chiapas. Cuadernos Universitarios, Serie Agronomía 1. Chapingo, México. 121 pp.

Lou, S. y C. L. Yurrita. 2005. Análisis de nicho alimentario en la comunidad de murciélagos frugívoros de Yaxhá, Petén, Guatemala. Acta Zool. Mex. 21(1): 83-94. 
Magurran, A. E. 1988. Ecological diversity and its measurement. Croom Helm. London, UK. 179 pp.

Maldonado-Sánchez, E. A. y F. Maldonado-Mares. 2010. Estructura y diversidad arbórea de una selva alta perennifolia en Tacotalpa, Tabasco, México. Universidad y Ciencia 26: $235-245$.

Manokaran, N. y J. V. LaFrankie. 1990. Stand structure of Pasoh Forest Reserve, a lowland rain forest in Peninsular Malaysia. J. Trop. For. Sci. 3: 14-24.

Martínez-Ramos, M. y X. García-Orth. 2007. Sucesión ecológica y restauración de las selvas húmedas. Bol. Soc. Bot. Méx. 80: 69-84.

Miranda, F. y E. Hernández X. 1963. Los tipos de vegetación de México y su clasificación. Bol. Soc. Bot. Méx. 28: 29-179.

Mueller-Dombois, D. y H. Ellenberg. 1974. Aims and methods of vegetation ecology. John Wiley. New York, USA. 547 pp.

Ochoa-Gaona, S. y G. Domínguez-Vázquez. 2000. Distribución y fenología de la flora leñosa de Chajul, Selva Lacandona, Chiapas, México. Brenesia 54: 1-24.

Ochoa-Gaona, S., F. Hernández-Vázquez, B. H. J. de Jong y F. Gurrí. 2007. Pérdida de diversidad florística ante un gradiente de intensificación del sistema agrícola de rozatumba-quema: un estudio de caso en la Selva Lacandona, Chiapas, México. Bol. Soc. Bot. Méx. 81: 65-80.

Ochoa-Gaona, S., C. Kampichler, B. H. J. de Jong, S. Hernández, V. Geissen y E. Huerta, 2010. A multi-criterion index for the evaluation of local tropical forest conditions in México. For. Ecol. Manag. 260: 618-627.

Orellana-Lara, J. 2009. Determinación de índices de diversidad florística arbórea en las parcelas permanentes de muestreo del Valle de Sacta. Tesis de Licenciatura. Escuela de Ciencias Forestales, Universidad Mayor de San Simón. Cochabamba, Bolivia. 49 pp. http://www.posgradoesfor.umss.edu.bo/boletin/umss/05\%20PASANTIAS/6\%20 pasantia.pdf (consultado el 22 de febrero de 2016).

Palacios, E. 2006. Ficha técnica de Bravaisia integerrima. Cuarenta y ocho especies de la flora de Chiapas incluidas en el PROY-NOM-059- ECOL-2000. Instituto de Historia Natural y Ecología. Bases de datos. SNIB CONABIO. Proyecto No. W008. México. D.F., México. http://www.conabio.gob.mx/conocimiento/ise/fichasnom/ Bravaisiaintegerrima00.pdf (consultado el 22 de febrero de 2016).

Palma, L. y J. Cisneros. 1996. Plan de uso sustentable de los suelos de Tabasco. Ed. Fundación PRODUCE Tabasco A.C. Villahermosa, México. 258 pp.

Pennington, T. D. y J. Sarukhán. 2005. Árboles tropicales de México. 2a. edición, Instituto de Ecología, Universidad Autónoma de México. Fondo de Cultura Económica. México, D.F., México. 521 pp.

Pérez, A., M. Sousa, A. M. Hannan, F. Chiang y P. Tenorio. 2005. Vegetación terrestre. In: Bueno, J., F. Álvarez y S. Santiago (eds.). Biodiversidad del estado de Tabasco. Instituto de Biología, Universidad Nacional Autónoma de México. Comisión Nacional para el Conocimiento y Uso de la Biodiversidad. México, D.F., México. pp. 65-110.

Pérez, L. A. y J. Sarukhán K. 1970. La vegetación de la región de Pichucalco, Chiapas. Publicación Especial No. 5. Comisión de Estudios sobre la Ecología de Dioscóreas. Instituto Nacional de Investigaciones Forestales. México, D.F., México. pp. 51-123. 
PMC. 2011. Manual de campo para el inventario forestal estatal cuantitativo. Bajo la coordinación de Fernando Paz; con la colaboración de Marcos Casiano, Carlos Omar Cruz, Jesús Argumedo, Ben de Jong y Rafael Flores. Programa Mexicano del Carbono. México, D.F., México. 129 pp.

Quinto-Mosquera, H., R. Rengifo-Ibargüen y Y. A. Ramos-Palacios. 2009. Mortalidad y reclutamiento de árboles en un bosque pluvial tropical de Chocó (Colombia). Rev. Fac. Nal. Agron. 62(1): 4855-4868.

Ramírez-González, A. 2006. Ecología: Métodos de muestreo y análisis de poblaciones y comunidades. Pontificia Universidad Javeriana. Bogotá, Colombia. 273 pp.

Ramos-Reyes, R., E. A. Maldonado-Sánchez y S. Ochoa Gaona. 2011. Análisis antropogénico de los cambios de usos, en dos reservas ecológicas en Tabasco, México. Agricultura Sostenible Vol. 7, CD. 10 pp.

Rzedowski, J. 1978. Vegetación de México. Ed. Limusa. México D.F., México. 432 pp.

Salazar-Conde, E. C., J. Zavala-Cruz, O. Castillo-Acosta y R. Cámara-Artigas. 2004. Evaluación espacial y temporal de la vegetación de la Sierra Madrigal, Tabasco, México (1973-2003). Investigaciones Geográficas 54: 7-23.

Sánchez-Munguía, A. 2005. Uso del suelo agropecuario y deforestación en Tabasco 19502000. Serie José Narciso Rovirosa. División Académica de Ciencias Biológicas, Universidad Juárez Autónoma de Tabasco. Villahermosa, México. 23 pp.

SARH. 1994. Inventario Nacional Forestal Periódico 1992-1994. Subsecretaria Forestal y de Fauna Silvestre. Secretaria de Agricultura y Recursos Hidráulicos. México D.F., México. $81 \mathrm{pp}$.

Semarnat. 2010. Norma Oficial Mexicana NOM-059-SEMARNAT-2010, Protección ambiental-Especies nativas de México de flora y fauna silvestres-Categorías de riesgo y especificaciones para su inclusión, exclusión o cambio-Lista de especies en riesgo, publicada en el Diario Oficial de la Federación el 30 de diciembre de 2010. Secretaría del Medio Ambiente y Recursos Naturales. México D.F., México. 78 p. http:// biblioteca.semarnat.gob.mx/janium/Documentos/Ciga/agenda/DOFsr/DO2454.pdf

Sol-Sánchez, A., A. Pérez-Vázquez, M. Vázquez-Torres, O. Ruiz-Rosado y O. CastilloAcosta. 1999. Diversidad y composición florística de la selva de canacoite en la Chontalpa, Tabasco, México. Universidad y Ciencia 15: 53-83.

Sollins, P. 1998. Factors influencing species composition in tropical lowland rain forest: does soil matter? Ecology 79: 23-30.

Tudela, F. 1989. La modernización forzada del trópico: el caso Tabasco, Proyecto integrado del Golfo. El Colegio de México-Centro de Investigaciones y de Estudios Avanzados del Instituto Politécnico Nacional-Federación Internacional de Institutos de Estudios Avanzados-Instituto de Investigaciones de las Naciones Unidas para el Desarrollo Social. México, D.F., México. 475 pp.

Tun-Dzul, F. J., H. Vester, R. Durán-García y B. Schmook. 2008. Estructura arbórea y variabilidad temporal del NDVI en los bajos inundables de la Península de Yucatán, México. Polibotánica 25: 69-90.

van Gemerden, B. S., H. Olff, M. P. E. Parren y F. Bongers. 2004. The pristine rain forest? Remnants of historical human impacts on current tree species composition and diversity. J. Biogeogr. 30: 1381-1390. 
Vázquez-Negrín, I., O. Castillo-Acosta, J. I. Valdez-Hernández, J. Zavala-Cruz y J. L. Martínez-Sánchez. 2011. Estructura y composición florística de la selva alta perennifolia en el ejido Niños Héroes Tenosique, Tabasco, México. Polibotánica 32: 41-61.

Zamora-Crescencio, P., G. García-Gil, J. S. Flores-Guido y J. Javier-Ortíz. 2008. Estructura y composición florística de la selva mediana subcaducifolia en el sur del estado de Yucatán, México. Polibotánica 26: 39-66.

Zarco-Espinoza, V. M., J. I. Valdez-Hernández, G. Ángeles-Pérez y O. Castillo-Acosta. 2010. Estructura y diversidad de la vegetación arbórea del Parque Estatal Agua Blanca, Macuspana, Tabasco. Universidad y Ciencia 26: 1-17. 


\section{APÉNDICE 1}

Lista de especies y valores de importancia relativa (VIR) de los árboles registrados en la selva mediana perennifolia de canacoite en el sitio Reserva Ecológica YCAH. Nomenclatura con base en The Plant List: http://www.theplantlist.org/ A: amenazada; P: en peligro de extinción (NOM-059-SEMARNAT-2010). Estrato que suelen alcanzar los adultos de la especie. $\mathrm{D}=$ densidad en el sitio (ind ha $\left.{ }^{-1}\right) ; \mathrm{AB}=$ área basal en el sitio $\left(\mathrm{m}^{2} \mathrm{ha}^{-1}\right) ; \mathrm{F}=$ frecuencia (no de $\left.10 \mathrm{UM}\right)$; VIR = valor de importancia relativo en $\%$.

\begin{tabular}{|c|c|c|c|c|c|c|}
\hline Especie & Familia & Estrato & $\mathrm{D}$ & $\mathrm{AB}$ & $\mathrm{F}$ & VIR \\
\hline Bravaisia integerrima (Spreng.) Standl. ${ }^{\mathrm{A}}$ & Acanthaceae & Medio & 2240 & 104.13 & 10 & 17.64 \\
\hline Astrocaryum mexicanum Liebm. ex Mart. & Arecaceae & Bajo & 3800 & 13.45 & 6 & 13.09 \\
\hline Spondias mombin $\mathrm{L}$. & Anacardiaceae & Alto & 20 & 47.45 & 9 & 6.13 \\
\hline Guarea glabra Vahl & Meliaceae & Alto & 620 & 16.15 & 10 & 5.22 \\
\hline $\begin{array}{l}\text { Cryosophila stauracantha (Heynh.) R.J. } \\
\text { Evans }^{{ }^{A}}\end{array}$ & Arecaceae & Bajo & 900 & 7.42 & 5 & 4.21 \\
\hline Bursera simaruba (L.) Sarg. & Burseraceae & Alto & 230 & 21.21 & 7 & 3.97 \\
\hline Cordia collococca $\mathrm{L}$. & Boraginaceae & Medio & 270 & 8.15 & 9 & 3.32 \\
\hline Ceiba pentandra (L.) Gaertn. & Malvaceae & Alto & 20 & 30.85 & 2 & 3.22 \\
\hline Licania platypus (Hemsl.) Fritsch & Chrysobalanaceae & Alto & 200 & 7.89 & 8 & 2.89 \\
\hline Ampelocera hottlei (Standl.) Standl. & Ulmaceae & Bajo & 440 & 3.74 & 6 & 2.79 \\
\hline Vatairea lundellii (Standl.) Record ${ }^{\mathrm{P}}$ & Leguminosae & Alto & 10 & 16.45 & 4 & 2.31 \\
\hline Simira salvadorensis (Standl.) Steyerm. & Rubiaceae & Alto & 440 & 3.35 & 3 & 2.14 \\
\hline Diospyros nigra (J.F. Gmel.) Perrier & Ebenaceae & Medio & 60 & 12.60 & 4 & 2.11 \\
\hline Pachira aquatica Aubl. & Malvaceae & Medio & 30 & 5.98 & 7 & 2.04 \\
\hline Lonchocarpus sp. & Leguminosae & Medio & 140 & 3.89 & 5 & 1.76 \\
\hline Guatteria anomala R.E. Fr. ${ }^{\mathrm{A}}$ & Annonaceae & Alto & 50 & 8.01 & 4 & 1.67 \\
\hline Swietenia macrophylla King & Meliaceae & Alto & 100 & 10.06 & 2 & 1.59 \\
\hline Casearia sp. & Salicaceae & Bajo & 250 & 2.95 & 3 & 1.58 \\
\hline Guarea sp. & Meliaceae & Alto & 110 & 2.69 & 5 & 1.57 \\
\hline Andira inermis (Wright) DC. & Leguminosae & Medio & 80 & 2.09 & 5 & 1.43 \\
\hline Pseudolmedia sp. & Moraceae & Medio & 300 & 1.31 & 2 & 1.37 \\
\hline Calophyllum brasiliense Cambess. ${ }^{\mathrm{A}}$ & Clusiaceae & Alto & 30 & 6.32 & 3 & 1.26 \\
\hline Platymiscium yucatanum Standl. & Leguminosae & Alto & 20 & 6.61 & 3 & 1.26 \\
\hline $\begin{array}{l}\text { Pleuranthodendron lindenii (Turcz.) } \\
\text { Sleumer }\end{array}$ & Salicaceae & Bajo & 140 & 1.23 & 3 & 1.11 \\
\hline
\end{tabular}


Apéndice 1. Continuación.

\begin{tabular}{|c|c|c|c|c|c|c|}
\hline Especie & Familia & Estrato* & $\mathrm{D}$ & $\mathrm{AB}$ & $\mathrm{F}$ & VIR \\
\hline Trichilia havanensis Jacq. & Meliaceae & Bajo & 10 & 1.61 & 4 & 0.98 \\
\hline Albizia saman (Jacq.) Merr. & Leguminosae & Medio & 10 & 2.60 & 3 & 0.87 \\
\hline Ormosia macrocalyx Ducke $^{\mathrm{P}}$ & Leguminosae & Alto & 200 & 0.75 & 1 & 0.83 \\
\hline Acacia cornigera (L.) Willd. & Leguminosae & Bajo & 120 & 0.85 & 2 & 0.82 \\
\hline Pouteria campechiana (Kunth) Baehni & Sapotaceae & Bajo & 20 & 1.28 & 3 & 0.78 \\
\hline Casearia corymbosa Kunth & Salicaceae & Bajo & 110 & 0.62 & 2 & 0.77 \\
\hline Tabernaemontana alba Mill. & Apocynaceae & Bajo & 60 & 1.30 & 2 & 0.69 \\
\hline Adelia barbinervis Cham. \& Schltdl. & Euphorbiaceae & Bajo & 100 & 0.76 & 1 & 0.55 \\
\hline Hampea macrocarpa Lundell & Malvaceae & Bajo & 100 & 0.72 & 1 & 0.55 \\
\hline Alibertia edulis (Rich.) A. Rich. ex DC. & Rubiaceae & Bajo & 100 & 0.67 & 1 & 0.54 \\
\hline $\begin{array}{l}\text { Amphitecna donnell-smithii (Sprague) } \\
\text { L.O. Williams }\end{array}$ & Bignoniaceae & Bajo & 100 & 0.62 & 1 & 0.54 \\
\hline Ficus sp. 1 & Moraceae & Medio & 10 & 0.35 & 1 & 0.52 \\
\hline Zygia cognata (Schltdl.) Britton \& Rose & Leguminosae & Medio & 30 & 2.58 & 1 & 0.52 \\
\hline $\begin{array}{l}\text { Tabebuia rosea (Bertol.) Bertero ex } \\
\text { A.DC. }\end{array}$ & Bignoniaceae & Medio & 80 & 0.62 & 1 & 0.48 \\
\hline Trophis racemosa (L.) Urb. & Moraceae & Medio & 70 & 0.42 & 1 & 0.44 \\
\hline Rollinia mucosa (Jacq.) Baill. & Annonaceae & Bajo & 50 & 0.89 & 1 & 0.42 \\
\hline Cojoba haematoloba L. Rico & Leguminosae & Medio & 10 & 1.54 & 1 & 0.37 \\
\hline Blepharidium guatemalense Standl. & Rubiaceae & Bajo & 10 & 1.45 & 1 & 0.36 \\
\hline Dialium guianense (Aubl.) Sandwith & Leguminosae & Alto & 10 & 1.39 & 1 & 0.36 \\
\hline Quararibea funebris (La Llave) Vischer & Malvaceae & Bajo & 10 & 1.39 & 1 & 0.36 \\
\hline Poulsenia armata (Miq.) Standl. & Moraceae & Alto & 40 & 0.34 & 1 & 0.35 \\
\hline Cecropia obtusifolia Bertol. & Urticaceae & Bajo & 20 & 0.61 & 1 & 0.31 \\
\hline Ocotea sp. & Lauraceae & Bajo & 20 & 0.48 & 1 & 0.30 \\
\hline Chrysophyllum mexicanum Brandegee & Sapotaceae & Bajo & 20 & 0.32 & 1 & 0.29 \\
\hline Coccoloba barbadensis Jacq. & Polygonaceae & Bajo & 20 & 0.19 & 1 & 0.28 \\
\hline Faramea occidentalis (L.) A. Rich. & Rubiaceae & Bajo & 20 & 0.24 & 1 & 0.28 \\
\hline Inga punctata Willd. & Leguminosae & Bajo & 10 & 0.51 & 1 & 0.28 \\
\hline Ficus sp. 2 & Moraceae & Medio & 10 & 3.18 & 1 & 0.26 \\
\hline Posoqueria sp. & Rubiaceae & Bajo & 10 & 0.21 & 1 & 0.25 \\
\hline
\end{tabular}




\section{APÉNDICE 2}

Lista de especies y valores de importancia relativa (VIR) de los árboles registrados en la selva mediana perennifolia de canacoite en el sitio Parque Estatal La Chontalpa PECH. Nomenclatura con base en The Plant List: http://www.theplantlist. org/. A: amenazada; P: en peligro de extinción (NOM-059-SEMARNAT-2010). Estrato que suelen alcanzar los adultos de la especie. $\mathrm{D}=$ densidad en el sitio (ind ha- ${ }^{1}$ ); $\mathrm{AB}=$ área basal en el sitio $\left(\mathrm{m}^{2} \mathrm{ha} \mathrm{a}^{-1}\right) ; \mathrm{F}=$ frecuencia (no de $\left.10 \mathrm{UM}\right) ; \mathrm{IVI}=$ valor de importancia (\%).

\begin{tabular}{|c|c|c|c|c|c|c|}
\hline Especie & Familia & Estrato & $\mathrm{D}$ & $\mathrm{AB}$ & $\mathrm{F}$ & IVI \\
\hline Bravaisia integerrima (Spreng.) Standl. ${ }^{\mathrm{A}}$ & Acanthaceae & Medio & 2210 & 80.32 & 9 & 18.92 \\
\hline Ficus sp. 2 & Moraceae & Media & 130 & 35.98 & 8 & 6.50 \\
\hline Spondias mombin L. & Anacardiaceae & Alto & 220 & 30.55 & 9 & 6.44 \\
\hline Faramea occidentalis (L.) A. Rich. & Rubiaceae & Bajo & 1260 & 4.66 & 6 & 6.37 \\
\hline Cordia collococca $\mathrm{L}$. & Boraginaceae & Medio & 530 & 9.12 & 9 & 5.10 \\
\hline Guazuma ulmifolia Lam. & Malvaceae & Medio & 320 & 26.32 & 4 & 5.07 \\
\hline $\begin{array}{l}\text { Pithecellobium lanceolatum (Willd.) } \\
\text { Benth. }\end{array}$ & Leguminosae & Medio & 500 & 11.95 & 8 & 5.06 \\
\hline Tabernaemontana alba Mill. & Apocynaceae & Bajo & 800 & 4.33 & 7 & 4.99 \\
\hline Pachira aquatica Aubl. & Malvaceae & Medio & 220 & 16.80 & 9 & 4.89 \\
\hline $\begin{array}{l}\text { Pleuranthodendron lindenii (Turcz.) } \\
\text { Sleumer }\end{array}$ & Salicaceae & Bajo & 730 & 4.50 & 6 & 4.52 \\
\hline Trichilia havanensis Jacq. & Meliaceae & Bajo & 520 & 4.00 & 7 & 3.99 \\
\hline Coccoloba barbadensis Jacq. & Polygonaceae & Bajo & 210 & 7.42 & 5 & 2.80 \\
\hline Trophis racemosa (L.) Urb. & Moraceae & Bajo & 280 & 2.97 & 4 & 2.30 \\
\hline Calophyllum brasiliense Cambess. ${ }^{\mathrm{A}}$ & Clusiaceae & Alto & 70 & 11.29 & 3 & 2.26 \\
\hline Vatairea lundellii (Standl.) Record ${ }^{\mathrm{P}}$ & Leguminosae & Alto & 60 & 10.67 & 3 & 2.16 \\
\hline Salacia elliptica (Mart.) G. Don & Celastraceae & Bajo & 260 & 2.41 & 3 & 1.92 \\
\hline Bursera simaruba (L.) Sarg. & Burseraceae & Alto & 90 & 5.95 & 3 & 1.73 \\
\hline $\begin{array}{l}\text { Attalea butyracea (Mutis ex L. f.) Wess. } \\
\text { Boer }\end{array}$ & Arecaceae & Bajo & 30 & 3.25 & 3 & 1.22 \\
\hline Zygia cognata (Schltdl.) Britton \& Rose & Leguminosae & Medio & 40 & 2.49 & 3 & 1.16 \\
\hline Acacia cornigera (L.) Willd. & Leguminosae & Bajo & 120 & 0.94 & 2 & 1.02 \\
\hline $\begin{array}{l}\text { Zanthoxylum riedelianum subsp. } \\
\text { kellermanii (P. Wilson) Reynel ex C. } \\
\text { Nelson }\end{array}$ & Rutaceae & Alto & 110 & 1.07 & 2 & 1.00 \\
\hline Brosimum alicastrum $\mathrm{Sw}$. & Moraceae & Alto & 110 & 0.99 & 2 & 0.99 \\
\hline
\end{tabular}


Apéndice 2. Continuación.

\begin{tabular}{llccccc}
\hline Especie & Familia & Estrato* & D & AB & F & IVI \\
\hline Sapindus saponaria L. & Sapindaceae & Bajo & 110 & 0.99 & 2 & 0.99 \\
Tabebuia rosea (Bertol.) Bertero ex A.DC. & Bignoniaceae & Medio & 60 & 2.40 & 2 & 0.98 \\
Nectandra ambigens (S.F. Blake) C. K. & Lauraceae & Bajo & 120 & 1.23 & 1 & 0.80 \\
$\quad$ & & & & & & \\
Allen & Rundia armata (Sw.) DC. & Bajo & 120 & 0.49 & 1 & 0.72 \\
Andira inermis (Wright) DC. & Leguminosae & Medio & 20 & 0.96 & 2 & 0.67 \\
Hampea nutricia Fryxell & Malvaceae & Bajo & 100 & 0.67 & 1 & 0.67 \\
Dendropanax arboreus (L.) Decne. \& & Araliaceae & Medio & 100 & 0.54 & 1 & 0.65 \\
$\quad$ Planch. & & & & & & \\
Brosimum sp. & Moraceae & Alto & 10 & 3.18 & 1 & 0.64 \\
Cecropia obtusifolia Bertol. & Urticaceae & Bajo & 80 & 0.82 & 1 & 0.62 \\
Ficus insipida Willd. & Moraceae & Medio & 10 & 1.67 & 1 & 0.47 \\
Inga sapindoides Willd. & Leguminosae & Bajo & 40 & 0.67 & 1 & 0.46 \\
Casearia corymbosa Kunth & Salicaceae & Bajo & 20 & 1.05 & 1 & 0.44 \\
Ficus citrifolia Mill. & Moraceae & Medio & 10 & 1.15 & 1 & 0.41 \\
Genipa americana L. & Rubiaceae & Bajo & 10 & 1.09 & 1 & 0.41 \\
Licania platypus (Hemsl.) Fritsch & Chrysobalanaceae & Alto & 10 & 0.50 & 1 & 0.34 \\
Lonchocarpus guatemalensis Benth. & Leguminosae & Medio & 20 & 0.18 & 1 & 0.34 \\
\hline & & & & &
\end{tabular}

